\title{
Thermodynamic Interpretation of Cryogenic Injection Experiments
}

\begin{tabular}{|r|l|}
\hline Journal: & $\begin{array}{l}\text { 47th AIAA/ASME/SAE/ASEE Joint Propulsion Conference \& Exhibit } \\
\text { 9th Annual International Energy Conversion Engineering Conference }\end{array}$ \\
\hline Manuscript ID: & Draft \\
\hline luMeetingID: & 2424 \\
\hline Date Submitted by the \\
Author: & n/a \\
\hline Contact Author: & Banuti, Daniel \\
\hline
\end{tabular}

\section{SCHOLARONE ${ }^{\text {Th }}$ \\ Manuscripts}




\title{
Thermodynamic Interpretation of Cryogenic Injection Experiments
}

\author{
Daniel T. Banuti* and Klaus Hannemann ${ }^{\dagger}$ \\ German Aerospace Center (DLR), Institute of Aerodynamics and Flow Technology, Göttingen, Germany
}

\begin{abstract}
This paper discusses a thermodynamic rather than mechanic discussion and interpretation of cryogenic injection of nitrogen in the vicinity of the critical point. There is no concensus in the literature on how to properly interpret and treat injection phenomena at supercritical pressures. While it is clear that the supercritical fluid loses many distinct liquid properties, such as heat of vaporization and surface tension, flows are being treated like they were liquid. Liquid core lengths are being determined in experiments, distinct droplets are tracked in computational fluid dynamic studies. And in fact, these approaches prove to be very successful. Nevertheless, a more appropriate treatment is desireable, taking into account the specifics of supercritical fluids. A contribution is attempted in this paper. The concept of pseudo-boiling, a maximum in heat capacity associated with a strong increase in specific volume, is discussed. It will be shown that the ensemble of supercritical maximum heat capacity states is in fact an extension to the saturation curve. A novel interpretation of the Clapeyron equation of thermodynamics in the limit of the critical point and beyond will be given. It will be shown that this generalization is able to characterize the pseudo-boiling line. Furthermore it will be shown that the slope $(\mathrm{d} \log p / \mathrm{d} T)$ is constant for supercritical conditions and equals the value at the critical point. The pseudo-boiling approach is then applied to characterize injection experiments. It can be shown that the power needed to reach the pseudo-boiling state correlates with the structure of the axial density distribution.
\end{abstract}

\section{Introduction}

Cryogenic propellants are a common sight among today's space propulsion systems. Cryogenic oxygen and hydrogen are used to fuel main stage engines (such as the European Vulcain II or the American Space Shuttle Main Engine) as well as upper stage engines (like the European Vinci). In these engines, liquid hydrogen is heated by regeneratively cooling the combustion chamber before being injected as a gas. Oxygen on the other hand remains cryogenic upon entering the chamber. Typically, for this propellant combination coaxial injectors are employed where a stream of cold, dense oxygen is surrounded by a stream of gaseous hydrogen. The exact thermodynamic state of the oxygen is dependent upon the combustion chamber pressure and injection temperature and strongly influences the atomization behavior.

Fundamental theoretical and numerical analysis of injection and combustion of cryogenic propellants have been carried out by Oefelein and Yang ${ }^{9}$ and Yang. ${ }^{14}$ Furthermore, in order to study these phenomena isolated from the complications of reactive flow in a real high pressure engine environment, inert model experiments are the accepted method of choice. Oschwald et al. ${ }^{10}$ give a thorough overview of these experiments. However, detailed data are scarce. Some of the best documented experiments on inert cryogenic injection have been carried out by Mayer et al. ${ }^{8}$ and Branam et al. ${ }^{2}$ Shadowgraphs, density distributions and temperatures are published of cryogenic nitrogen injection into a high pressure nitrogen environment. They report that the jet does not resemble a liquid jet. With particular importance to numerical modeling of rocket engine injection, no droplets have been observed. The stream instead behaves like a gaseous jet albeit with liquid like densities.

\footnotetext{
${ }^{*}$ Research Engineer and PhD Student, German Aerospace Center (DLR), Institute of Aerodynamics and Flow Technology Spacecraft Section, Göttingen, Bunsenstr. 10, Germany.

${ }^{\dagger}$ Head of Spacecraft Section, German Aerospace Center (DLR), Institute of Aerodynamics and Flow Technology, Göttingen, Bunsenstr. 10, Germany, Member AIAA.
} 
Multiphase terminology and concepts are typically used to characterize flow in the vicinity of the critical point. Droplet models are employed successfully despite a lack of surface tension. Jet mixing is discussed in terms of atomization, primary and secondary break-up, and vaporization. Estimation of the 'liquid core length' is often used to characterize such flows despite the unclarity concerning the presence of a liquid phase during this process. In fact, CFD, ranging from the original papers of Mayer and Branam (RANS) to the recent publications of Schmitt et al. ${ }^{11}$ (LES) and Kim et al. ${ }^{6}$ (RANS), predicts a region of constant high density right after the injector. This appears to supports the notion of a liquid core despite the supercritical thermodynamic state - but it is contrary to experimental findings. Only two out of eight published axial density distributions of Mayer and Branam exhibit a constant density core. A dense core is hence merely a special case of supercritical injection. The authors of this paper ${ }^{1}$ suggest that the jet density decay is a thermodynamic rather than a mechanical process, resembling vaporization rather than atomization. In fact, it appears that injector wall heat flux can prevent the jet from showing a constant density core in the injection region by accelerating the vaporization-like process. This is indicated by numerical computations using the DLR TAU code (Gerhold et al., ${ }^{4}$ Mack et al. ${ }^{7}$ ) extended by a real gas equation of state treatment by Bartolome Calvo and Hannemann. ${ }^{3}$

The purpose of this paper is to extend this previous study by introducing a quantitative thermodynamic interpretation of supercritical pseudo-boiling, as discussed by Oschwald et al. ${ }^{10}$ The Clapeyron equation of phase equilibrium will be interpreted for supercritical states. This approach is then applied to interpret and characterize the nitrogen injection experiments of Mayer quantitatively. Especially a correlation between thermodynamic state and axial density distribution will be discussed.

\section{Background}

\section{II.A. Nitrogen Injection Experiment}

Starting point of this study was the analysis of nitrogen injection experiments by Mayer et al. ${ }^{8}$ and Branam et al. ${ }^{2}$

A detailed description of the experiment has been given by Branam. ${ }^{2}$ In their experiments, cryogenic nitrogen is injected at supercritical pressures into a chamber filled with nitrogen at room temperature. Axial and radial density profiles, core length, jet spreading angles, length scales and shadowgraphs are published for $4 \mathrm{MPa}, 5 \mathrm{MPa}$, and $6 \mathrm{MPa}$ chamber pressure, $100 \mathrm{~K}, 120 \mathrm{~K}$, and $130 \mathrm{~K}$ fluid temperature at $5 \mathrm{~m} / \mathrm{s}$ and $2 \mathrm{~m} / \mathrm{s}$ injection velocity. Test cases are compiled in Table 1, case numbering follows Mayer. The temperature $\mathrm{T} 1$ is measured upstream in the injector, i.e. $90 \mathrm{~mm}$ upstream of the injector exit. A thermocouple of $1 \mathrm{~mm}$ diameter is introduced into the free jet in two positions: axially, reaching $1 \mathrm{~mm}$ inside the injector (temperature $\mathrm{Tb}_{i n j}$ ), and radially, $1 \mathrm{~mm}$ downstream of the injector exit (temperature $\mathrm{Ta}_{i n j}$ ). The thermodynamic state of these experiments is chosen to be close to the critical point of nitrogen. This results in a sensitive dependence of density on temperature, as can be seen in Fig. 1(a). Solid lines represent real gas data, taken from the NIST database, based on Span et al., ${ }^{12}$ dotted lines correspond to ideal gas data. RCM1a and RCM1b correspond to cases 3 and 11, respectively, and are often used test cases as defined in the Rocket Combustion Modeling Workshop 2001. ${ }^{5}$ Published axial density distributions are shown in Fig. 1(b).

\section{II.B. Pseudo Boiling}

The term 'pseudo-boiling', e.g. as used by Oschwald et al., ${ }^{10}$ denotes the extension of the saturated pressure curve into supercritical states. At subcritical pressures, the heat of vaporization needs to be added for a substance to change its state through the saturated pressure curve. Approaching the critical point, the heat of vaporization vanishes. Nevertheless, when isobarically heating a substance above the critical point, a distinct peak in specific heat can be found which needs to be 'passed'. This can be seen in Fig. 2(a). The peak coincides with the region of maximum $\partial \rho / \partial T$ as can be seen in Fig. 1(a). Figure 2(b) shows the saturated vapor curve of nitrogen from the triple point to the critical point. Added are points of maximum $c_{p}$ for, from low to high pressures, 4, 5, 6, 7, 8, 9, $10 \mathrm{MPa}$. Data are taken from the NIST database, based on Span et al. ${ }^{12}$ Thus, when changing a state isobarically through the region of maximum specific heat, an energy $\int c_{p} \mathrm{~d} T$ has to be added and the substance's specific volume increases significantly. Hence the term 'pseudo-boiling', as it resembles subcritical vaporization. Eq. (1) quantifies the needed power $P_{\mathrm{V}}$ to heat a flow from the injection temperature $T_{\mathrm{in}}$ to the pseudo boiling temperature $T_{\mathrm{pb}}$ as the product of the required 
Table 1. Overview of test matrix from Mayer et al. ${ }^{8}$

\begin{tabular}{rccc}
\hline Case & $\begin{array}{c}p_{\mathrm{c}} \\
\text { in MPa }\end{array}$ & $\begin{array}{c}v_{1} \\
\text { in } \mathrm{m} / \mathrm{s}\end{array}$ & $\begin{array}{c}T \\
\text { in } \mathrm{K}\end{array}$ \\
\hline 1 & 4.0 & 2.0 & 120.0 \\
2 & 4.0 & 2.0 & 130.0 \\
3 & 4.0 & 5.0 & 120.0 \\
4 & 4.0 & 5.0 & 130.0 \\
5 & 5.0 & 2.0 & 120.0 \\
6 & 5.0 & 2.0 & 130.0 \\
7 & 5.0 & 5.0 & 120.0 \\
8 & 5.0 & 5.0 & 130.0 \\
9 & 6.0 & 2.0 & 120.0 \\
10 & 6.0 & 2.0 & 130.0 \\
11 & 6.0 & 5.0 & 120.0 \\
12 & 6.0 & 5.0 & 130.0 \\
\hline
\end{tabular}

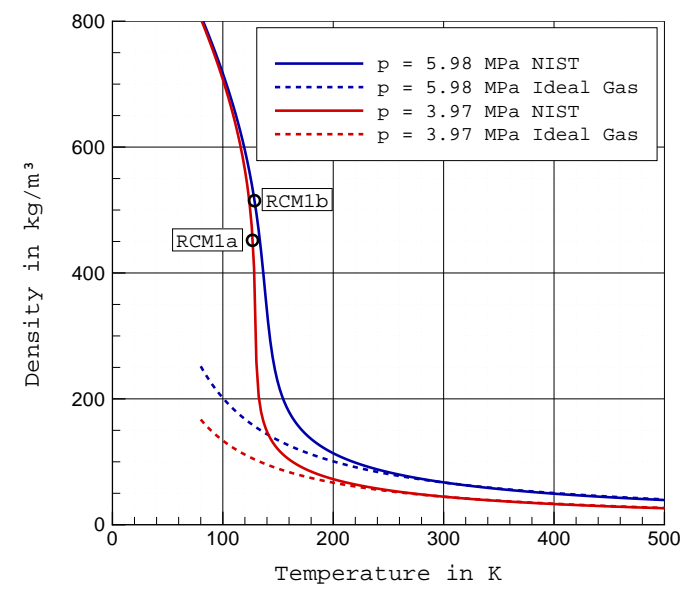

(a) Nitrogen isobaric density-temperature plot, real gas and ideal gas.

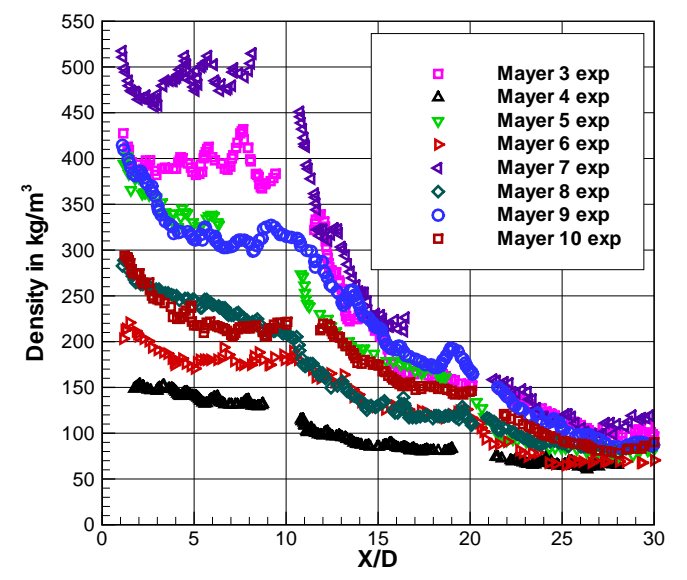

(b) Axial density distributions of nitrogen injection experiments from Mayer. ${ }^{8}$

Figure 1. Cryogenic nitrogen density at supercritical pressures.

energy $q_{\mathrm{V}}$ per unit mass and the massflux $\dot{m}$.

$$
P_{\mathrm{V}}=\dot{m} \cdot q_{\mathrm{V}}=\dot{m} \cdot \int_{T_{\mathrm{in}}}^{T_{\mathrm{pb}}} c_{p}(T) \mathrm{d} T=\rho \cdot u \cdot A \cdot \int_{T_{\mathrm{in}}}^{T_{\mathrm{pb}}} c_{p}(T) \mathrm{d} T
$$

\section{II.C. Clapeyron Equation}

Determination of a phase equilibrium is discussed in many textbook, see e.g. Weigand et al. ${ }^{13}$ With only one substance present, at thermal and mechanical equilibrium, equilibrium can be expressed as specific Gibbs free enthalpy equilibrium between states ' (liquid) and " (vapor)

$$
\mathrm{d} g^{\prime}=v^{\prime} \mathrm{d} p-s^{\prime} \mathrm{d} T=\mathrm{d} g^{\prime \prime}=v^{\prime \prime} \mathrm{d} p-s^{\prime \prime} \mathrm{d} T
$$

where $p, T, s$, and $v$ are pressure, temperature, specific entropy, and specific volume, respectively. This can be rearranged to find

$$
\frac{\mathrm{d} p}{\mathrm{~d} T}=\frac{s^{\prime \prime}-s^{\prime}}{v^{\prime \prime}-v^{\prime}}
$$




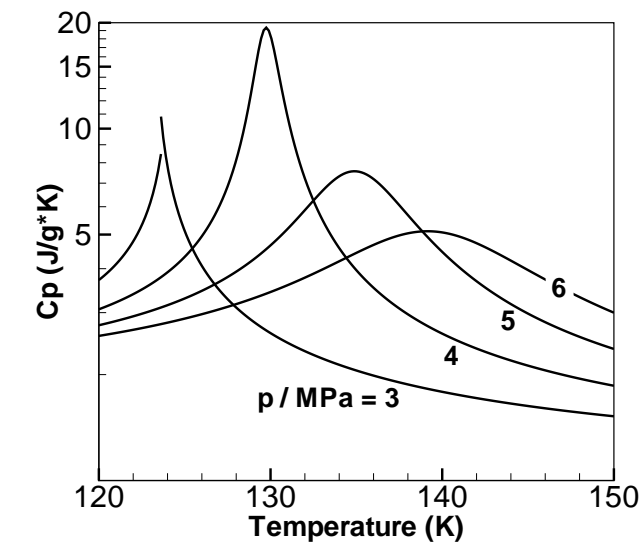

(a) Nitrogen specific heat capacity at subcritical (3 $\mathrm{MPa})$ and supercritical pressures (4..6 MPa).

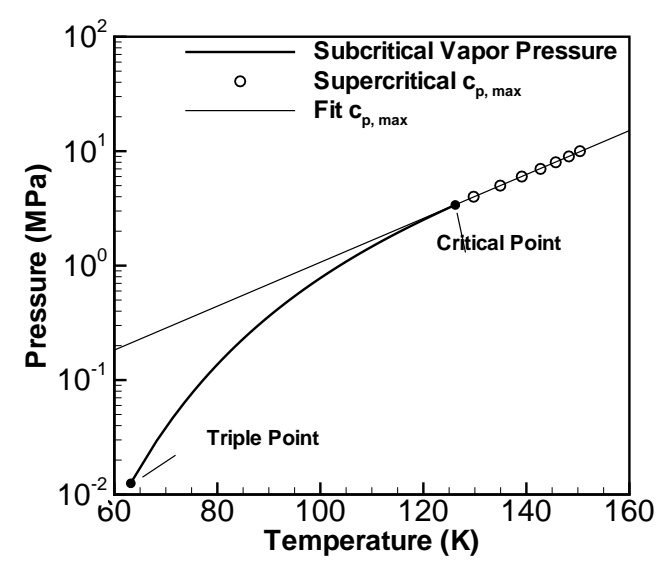

(b) Nitrogen vapor pressure curve and supercritical states of maximum specific heat capacity.

Figure 2. Behavior of nitrogen at the critical point.

Using $\Delta s=s^{\prime \prime}-s^{\prime}, \Delta v=v^{\prime \prime}-v^{\prime}$ and $\Delta s=\Delta h / T$, where $h$ is specific enthalpy, yields the Clapeyron equation

$$
\frac{\mathrm{d} p}{\mathrm{~d} T}=\frac{\Delta s}{\Delta v}=\frac{1}{T} \frac{\Delta h}{\Delta v}
$$

When applying Eq. (4) at the saturation curve

$$
\frac{\mathrm{d} p_{\mathrm{s}}}{\mathrm{d} T}=\frac{1}{T} \frac{\Delta h_{\mathrm{V}}}{\Delta v}
$$

$\Delta h_{\mathrm{V}}$ can now be interpreted as the heat of vaporization, $\Delta v$ is the increase in volume due to vaporization. Figure 3 shows how $s$ and $v$ change over a phase change for nitrogen at 3.2 and 3.35 MPa. Data are again taken from the NIST database, based on Span et al. ${ }^{12}$ On the left side, liquid values can be read off, on the right side are vapor data. It can be seen, how the difference decreases with growing pressure and vanishes as the supercritical pressure of $3.50 \mathrm{MPa}$ is reached.

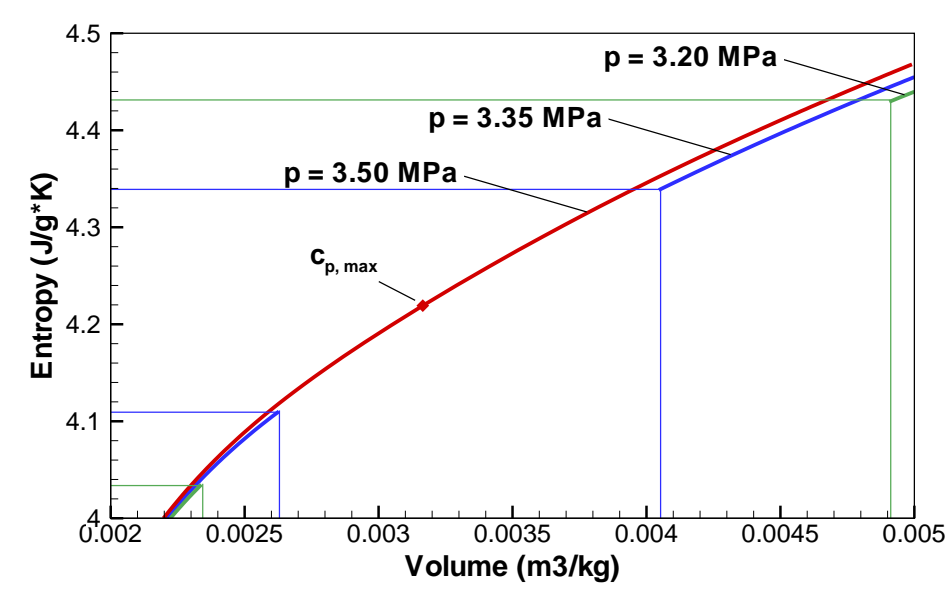

Figure 3. Change of specific entropy and volume at saturation curve. 


\section{Results}

\section{III.A. Supercritical Interpretation and Extension}

Figure 3 shows how $\Delta s$, i.e. $\Delta h_{\mathrm{V}} / T$, and $\Delta v$ decrease when the pressure is increased from $3.2 \mathrm{MPa}$ to 3.35 MPa. Approaching and exceeding the critical point, the process changes from discontinuous to continuous. The heat of vaporization $\Delta h_{\mathrm{V}}$ vanishes. No discontinuous increase in volume occurs anymore, i.e. $\Delta v$ also vanishes. In summary: the discontinuous phase change seizes to exist and is replaced by a continuous, gradual change in properties for isobaric heating at supercritical pressures. Yet, the concept of pseudo-boiling is plausible, Fig. 2(b) shows that the pseudo-boiling line is indeed a valid extension of the saturation curve. How can this be interpreted thermodynamically? How can the Clapeyron equation be evaluated when the heat of vaporization has vanished?

Watching $\Delta s$ and $\Delta v$ as the critical point is approached and passed, both approach zero, i.e. $\Delta s \rightarrow 0$ and $\Delta v \rightarrow 0$. While evaluating a zero $\Delta h_{\mathrm{V}}$ or $\Delta v$ alone does not make sense, evaluating the ratio of the two does. In fact, letting both differences approach zero is this is the mathematical process of obtaining a differential. Thus, approaching critical conditions

$$
\left(\frac{\mathrm{d} p}{\mathrm{~d} T}\right)_{s}=\left(\frac{\Delta s}{\Delta v}\right)_{s} \stackrel{p, T \rightarrow p_{\mathrm{cr}}, T_{\mathrm{cr}}}{\longrightarrow}\left(\frac{\partial s}{\partial v}\right)_{\mathrm{cr}}
$$

In the supercritical regime

$$
\left(\frac{\mathrm{d} p}{\mathrm{~d} T}\right)_{\mathrm{pb}}=\left(\frac{\partial s}{\partial v}\right)_{\mathrm{pb}}
$$

This can be readily applied in Fig. 3. It can be seen how $\Delta s$ and $\Delta v$ decrease towards the critical pressure of $3.39 \mathrm{MPa}$, the two curves for liquid and vapor merge into one upon exceeding the critical pressure as can be seen for 3.5 MPa. Figure 2(b) shows that the line of maximal heat capacities attaches smoothly to the saturation curve. Thus now everything is at hand to interpret the $3.5 \mathrm{MPa}$ curve in Fig. 3. The tangent at the curve in the point of maximum $c_{p}$ is the appropriate slope to extract from this data to describe the pseudo-boiling curve, Eq. (7).

Going back to Fig. 2(b) one more thing can be found: while the slope $(\mathrm{d} \log p / \mathrm{d} T)_{\mathrm{S}}$ is gradually decreasing in the subcritical region, it is constant upon entering a supercritical state and equal to the slope at the critical point.

$$
\left(\frac{\mathrm{d} \log p}{\mathrm{~d} T}\right)_{\mathrm{s}} \stackrel{T \rightarrow T_{\mathrm{cr}}}{\longrightarrow}\left(\frac{\mathrm{d} \log p}{\mathrm{~d} T}\right)_{\mathrm{cr}}=\left(\frac{\mathrm{d} \log p}{\mathrm{~d} T}\right)_{\mathrm{pb}}=\hat{\mathcal{C}}
$$

The equality is still valid for a logarithm of a different base, such as the natural logarithm, only the constant needs to be adapted.

$$
\left(\frac{\mathrm{d} \ln p}{\mathrm{~d} T}\right)_{\mathrm{pb}}=\left(\frac{\mathrm{d} \ln p}{\mathrm{~d} T}\right)_{\mathrm{cr}}=\mathcal{C}
$$

Rearranged

$$
\int_{p_{\mathrm{cr}}}^{p_{\mathrm{pb}}} \mathrm{d} \ln p=\int_{T_{\mathrm{cr}}}^{T_{\mathrm{pb}}} \mathcal{C} \mathrm{d} T
$$

this can be readily integrated

$$
p_{\mathrm{pb}}=p_{\mathrm{cr}} \exp \left[\mathcal{C}\left(T_{\mathrm{pb}}-T_{\mathrm{cr}}\right)\right]
$$

For nitrogen, as shown in Fig. 2(b), the constant is estimated to $\mathcal{C}=0.044$, critical pressure and temperature are $3.39 \mathrm{MPa}$ and $126.2 \mathrm{~K}$, respectively.

\section{III.B. Axial Density Distributions}

III.B.1. Analysis

Results of the cryogenic nitrogen injection experiments by Mayer et al. ${ }^{8}$ and Branam et al. ${ }^{2}$ have been shown in Fig. 1(b). In order to compare the density distributions, they have been nondimensionalized using the injection density $\rho_{\text {in }}$ and the farfield density $\rho_{\infty}$.

$$
\rho^{+}=\frac{\rho-\rho_{\infty}}{\rho_{\text {in }}-\rho_{\infty}}
$$

$$
5 \text { of } 9
$$


Nondimensionalized axial density distributions are then plotted, grouped by temperature and injection velocity in Fig. 4. Unfortunately, no density distribution data have been published for cases 1, 2, $11,12$. Now, two curves are plotted in each figure, for identical conditions except for the pressure. It can be seen

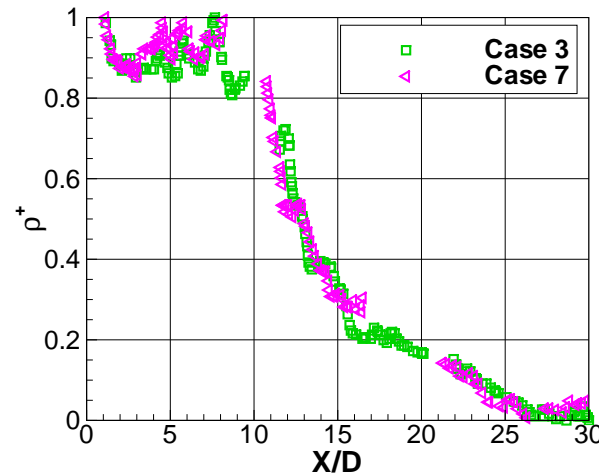

(a) $u=5 \mathrm{~m} / \mathrm{s}, T=120 \mathrm{~K}$

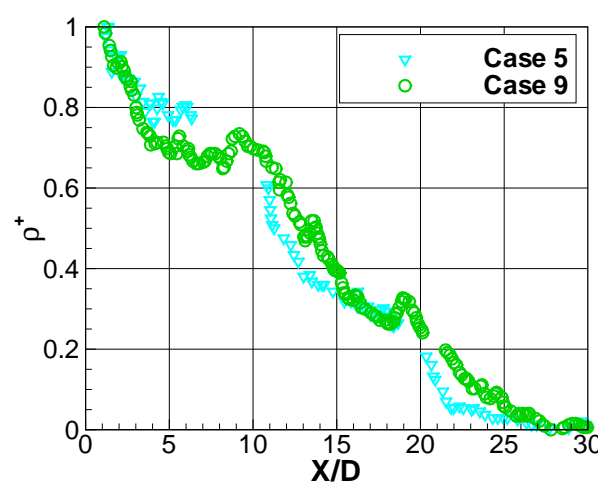

(c) $u=2 \mathrm{~m} / \mathrm{s}, T=120 \mathrm{~K}$

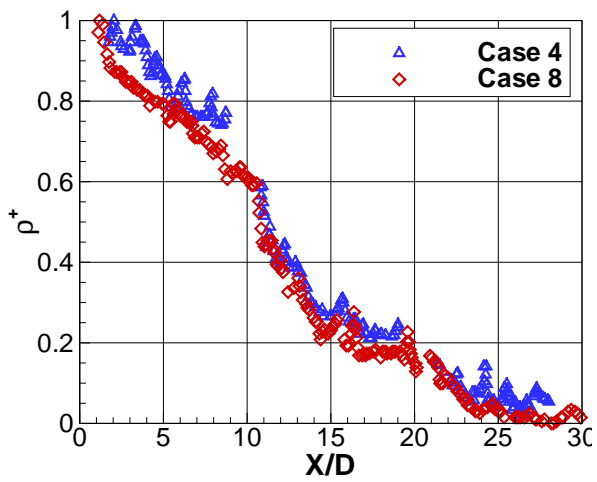

(b) $u=5 \mathrm{~m} / \mathrm{s}, T=130 \mathrm{~K}$

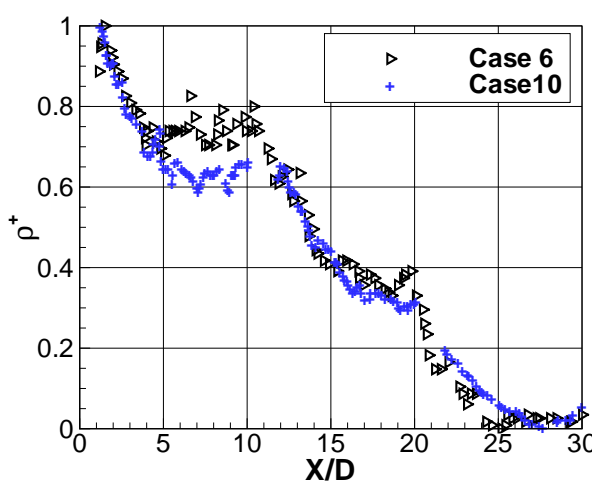

(d) $u=2 \mathrm{~m} / \mathrm{s}, T=130 \mathrm{~K}$

Figure 4. Nondimensional axial density distributions from Mayer. ${ }^{8}$

that the curves in each figure clearly resemble each other. Furthermore, a categorization can be carried out depending on the density distribution characteristics. Figure 4(a) shows a constant density core and will be dubbed type 'c'. The distributions in Fig. 4(b) exhibit a strictly monotonous slope, hence type 's'. Finally, Figs. 4(c) and 4(d) show distributions that decline right away, before reaching an intermediate plateau and will be dubbed type 'p'.

Carrying out an analysis using traditional multiphase methodology, one would expect a liquid core of constant density in each case. However, this is clearly not the case. How can this be explained? First, available data needs to be sighted. Data obtained in the experiments is compiled in Tab. 2, following Mayer's nomenclature, left of the vertical line. These are chamber pressure $p_{\mathrm{c}}$, inflow velocity $v_{1}$, temperature upstream in the injector $T 1$, temperature in injector exit measured normal to the flow, $T a_{\text {inj }}$, and parallel to the flow, $T b_{\text {inj. }}$. Derived data are added to the right of Tab. 2. These are the injection density, taken from the NIST database corresponding to $p_{\mathrm{c}}$ and $T 1$, pseudo-boiling temperature $T_{\mathrm{pb}}$ for the target pressure (see Tab. 1), pseudo-vaporization power $P_{\mathrm{V}, T a}$ according to Eq. (1) from $T a_{\mathrm{inj}}$ to $T_{\mathrm{pb}}$, and momentum flux $\dot{I}=v_{1} \dot{m}$ and density distribution type.

Given the lack of liquid properties at supercritical pressures, as discussed before in, ${ }^{1}$ the data will be interpreted in terms of mechanical interaction and thermodynamic state. If this is true, similarities between the axial density distributions should show up when they are grouped by temperature and injector residence time $^{\text {a }}$, i.e. velocity.

Momentum flux is plotted in Fig. 5 to compare the mechanical attributes of the different cases. And indeed, cases $5,6,9$, 10, which exhibit the same plateau behavior, appear with a comparable momentum flux.

aThis needs to be taken into account to allow for injector wall heat flux. 
Table 2. Measured values from Mayer et al., ${ }^{8} \mathrm{~T} 1$ from Branam et al. ${ }^{2}$ and derived data

\begin{tabular}{rccccc|cccccc}
\hline Case & $\begin{array}{c}p_{\mathrm{c}} \\
\text { in MPa }\end{array}$ & $\begin{array}{c}v_{1} \\
\text { in } \mathrm{m} / \mathrm{s}\end{array}$ & $\begin{array}{c}T 1 \\
\text { in K }\end{array}$ & $\begin{array}{c}T a_{\text {inj }} \\
\text { in K }\end{array}$ & $\begin{array}{c}T b_{\text {inj }} \\
\text { in K }\end{array}$ & $\begin{array}{c}\rho\left(p_{\mathrm{c}}, T 1\right) \\
\text { in } \mathrm{kg} / \mathrm{m}^{3}\end{array}$ & $\begin{array}{c}\dot{m} \\
\text { in } \mathrm{kg} / \mathrm{s}\end{array}$ & $\begin{array}{r}P_{\mathrm{V}, T a} \\
\text { in } / \mathrm{s}\end{array}$ & $\begin{array}{c}\dot{I} \\
\text { in kg m/s }\end{array}$ & $\begin{array}{c}T_{\mathrm{pb}} \\
\text { in K }\end{array}$ & Type \\
\hline 1 & 3.96 & 1.8 & 120.4 & 130.0 & 122.2 & 560.6 & $3.84 \mathrm{e}-3$ & -29.2 & $6.90 \mathrm{E}-03$ & 129.8 & - \\
2 & 3.95 & 2.7 & 129.4 & 140.0 & 131.9 & 349.1 & $3.58 \mathrm{e}-3$ & -219.2 & $9.67 \mathrm{E}-03$ & 129.8 & - \\
3 & 3.97 & 4.9 & 120.9 & 126.9 & 122.2 & 555.1 & $1.03 \mathrm{e}-2$ & 321.8 & $5.07 \mathrm{E}-02$ & 129.8 & $\mathrm{c}$ \\
4 & 3.98 & 5.4 & 130.7 & 137.0 & 133.3 & 258.9 & $5.31 \mathrm{e}-3$ & -279.5 & $2.87 \mathrm{E}-02$ & 129.8 & $\mathrm{~s}$ \\
5 & 4.90 & 2.0 & 120.7 & 131.0 & 122.6 & 575.5 & $4.37 \mathrm{e}-3$ & 114.4 & $8.75 \mathrm{E}-03$ & 134.9 & $\mathrm{p}$ \\
6 & 4.90 & 1.9 & 129.8 & 140.0 & 131.5 & 464.0 & $3.35 \mathrm{e}-3$ & -106.4 & $6.37 \mathrm{E}-03$ & 134.9 & $\mathrm{p}$ \\
7 & 5.01 & 4.5 & 120.7 & 126.2 & 122.5 & 575.5 & $9.84 \mathrm{e}-3$ & 454.5 & $4.43 \mathrm{E}-02$ & 134.9 & $\mathrm{c}$ \\
8 & 5.00 & 4.9 & 130.2 & 135.7 & 131.7 & 456.6 & $8.50 \mathrm{e}-3$ & -57.4 & $4.17 \mathrm{E}-02$ & 134.9 & $\mathrm{~s}$ \\
9 & 5.85 & 2.0 & 120.7 & 135.0 & 122.8 & 589.7 & $4.48 \mathrm{e}-3$ & 91.4 & $8.97 \mathrm{E}-03$ & 139.2 & $\mathrm{p}$ \\
10 & 5.88 & 1.9 & 129.9 & 140.5 & 131.9 & 501.8 & $3.62 \mathrm{e}-3$ & -20.3 & $6.89 \mathrm{E}-03$ & 139.2 & $\mathrm{p}$ \\
11 & 5.98 & 4.9 & 121.7 & 128.7 & 123.3 & 581.7 & $1.08 \mathrm{e}-2$ & 652.6 & $5.31 \mathrm{E}-02$ & 139.2 & - \\
12 & 5.96 & 4.9 & 130.8 & 135.4 & 132.3 & 490.8 & $9.14 \mathrm{e}-3$ & 287.9 & $4.48 \mathrm{E}-02$ & 139.2 & - \\
\hline
\end{tabular}

However, no distinct difference can be seen between cases 7 and 8 whereas their density distributions are very different. Evaluating Eq. (1) for each of the cases shown in Table 2 yields the required power to reach

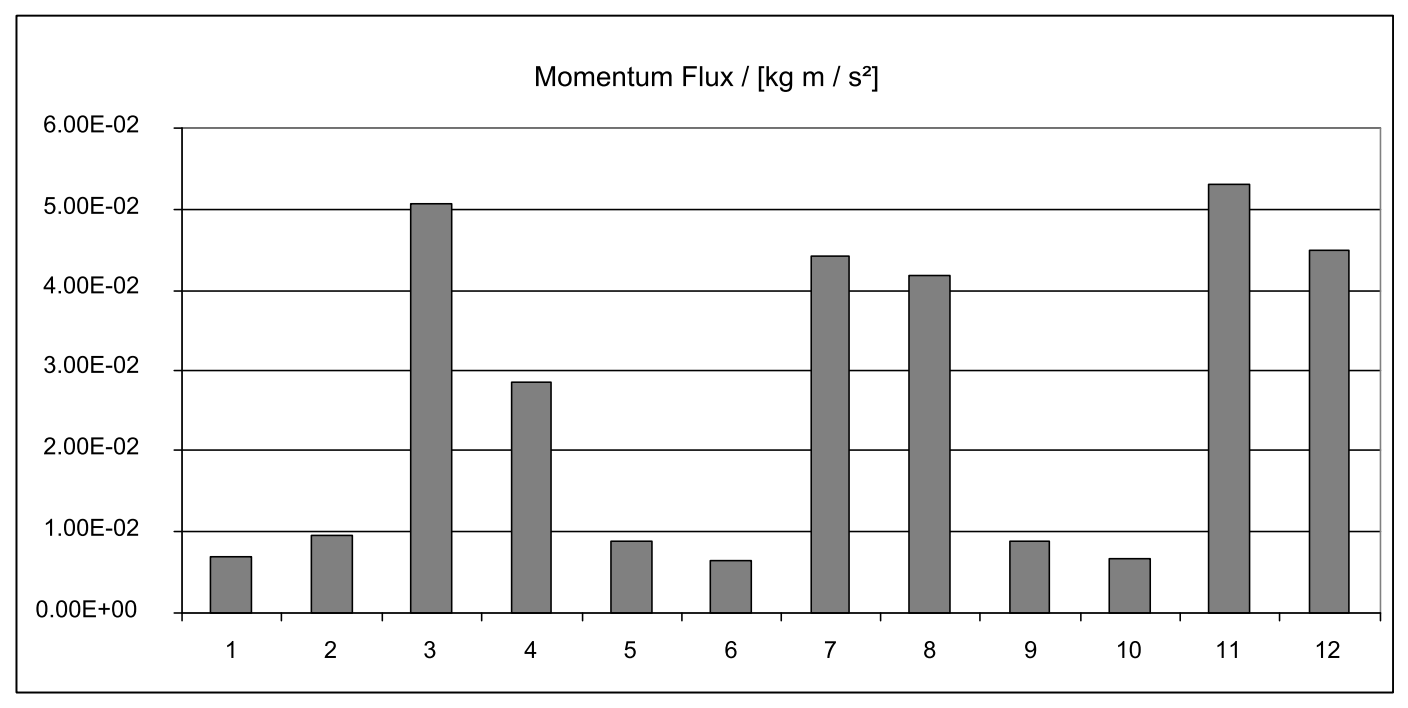

Figure 5. Momentum of jet emanating into chamber.

the pseudo-boiling state. The results are shown in Fig. 6. Here, cases 3 and 7 , which are the only ones exhibiting a dense core distribution, clearly stand out from the rest. Apparently, the dense core cases are also the cases which require the highest amount of pseudo-vaporization power.

\section{III.B.2. Interpretation}

Figure 4 reveals that the suggested grouping is indeed reasonable. In each figure, the axial distributions clearly show similarities to each other. Fig. 4(a) is the only group that exhibits a constant density core. Fig. 4(b) on the other hand shows a rapid decline of density from the injector exit. Figs. 4(c) and 4(d) actually resemble each other in that they show a density drop from the injector but reach a plateau before dropping further.

This can be interpreted using Fig. 6. Cases 3 and 7 in Fig. 4(a) are the ones with the by far highest required power to reach the pseudo-boiling state. Thus, it takes significant time until enough heat is transferred to the fluid to significantly reduce its density. Cases 5, 6, 9, 10 are already close to the pseudo-boiling point. Cases 5 and 6 have the highest power need which reflects on the higher density plateau. 


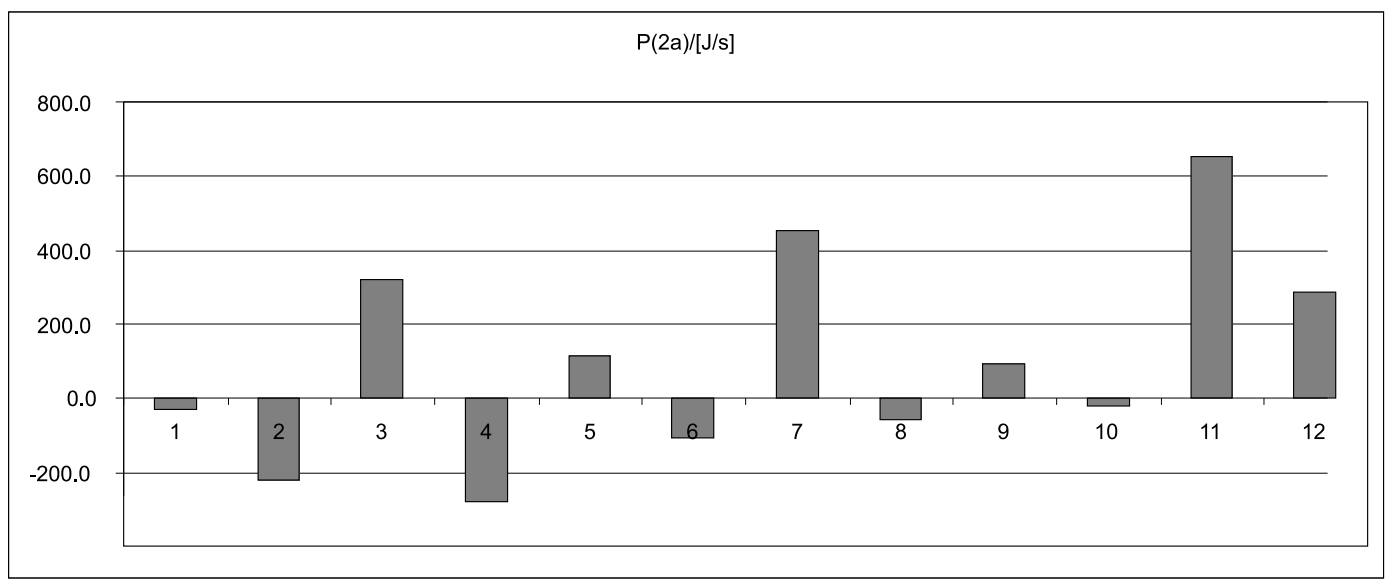

Figure 6. Power required to reach the pseudo-boiling state in steady flow.

Cases 5, 6, 9 , and 10 show a distinct similarity in the momentum flux plot Fig. 5 and in the density distribution Figs. 4(c) and 4(d). This suggests that a mechanical mechanism is dominant is these cases.

The power approach is not capable of differentiating between cases of low required power (e.g. slope case 8 is grouped between the plateau types 5 and 6 ), but clearly points out dense core types. The momentum approach is not capable of differentiating between high momentum cases (core type 7 and slope type 8 almost have the same value) but reflects similarity between the plateau cases 5, 6, 9, and 10 .

\section{Conclusion}

This paper has suggested a thermodynamic interpretation approach to supercritical fluid behavior, in particular cryogenic injection.

The concept of the Clapeyron equation could be generalized and applied to supercritical states. It could thus be shown that pseudo-boiling can be understood as a valid thermodynamic concept. The state can be described using the extended Clapeyron ansatz. It is characterized by a maximum heat capacity of the fluid, along a large dependency of volume to temperature. It thus really resembles boiling, albeit in a continuous form. An equation has been introduced that encompasses all supercritical states of maximum specific heat capacity to a pseudo boiling line.

Injection experiments have been analyzed with regard to the occurrence of a high density core, as typically witnessed in subcritical injection. Three distinct density distributions have been identified, a dense core, a monotonous slope, and a plateau. Thus, the dense core is merely a special case in supercritical injection. A thermodynamic approach, regarding the power needed to reach the pseudo-boiling state, has been able to point out the dense core cases. This indicates a correlation between supercritical cryogenic jet evolution and their thermodynamic state. The plateau cases could best be characterized using the mechanical approach employing the momentum flux. Hence, for some cases, cryogenic jet disintegration resembles a pseudovaporization process more than a mechanical atomization.

\section{References}

${ }^{1}$ D.T. Banuti and K. Hannemann. Effect of injector wall heat flux on cryogenic injection. In Proceedings of the 46th AIAA/ASME/SAE/ASEE Joint Propulsion Conference and Exhibit, number AIAA-2010-7139, Nashville, USA, 2010. AIAA.

${ }^{2}$ R. Branam and W. Mayer. Characterization of cryogenic injection at supercritical pressure. Journal of Propulsion and Power, 19(3):342-355, 2003.

${ }^{3}$ J. Bartolome Calvo and K. Hannemann. Numerical simulation of liquid rocket engine cooling channels. In Proceedings of the 45th AIAA/ASME/SAE/ASEE Joint Propulsion Conference \&J Exhibit, Denver, 2009.

${ }^{4}$ T. Gerhold, M. Galle, O. Friedrich, and J. Evans. Calculation of complex three-dimensional configurations employing the DLR-TAU-code. In Proceedings of the 35th Aerospace Sciences Meeting and Exhibit, number AIAA-1997-167, Reno, USA, 1997. AIAA.

${ }^{5}$ Oskar J Haidn. Proceedings of the second international workshop rocket combustion modeling - atomization, combustion and heat transfer. Lampoldshausen, 2001.

${ }^{6}$ T. Kim, Y. Kim, and S.-K. Kim. Numerical study of cryogenic liquid nitrogen jets at supercritical pressures. The Journal 
of Supercritical Fluids, 56:152-163, 2011.

${ }^{7}$ A. Mack and V. Hannemann. Validation of the unstructured DLR-TAU-code for hypersonic flows. In Proceedings of the 32nd AIAA Fluid Dynamics Conference and Exhibit, number AIAA-2002-3111, 2002.

${ }^{8}$ W. Mayer, J. Telaar, R. Branam, G. Schneider, and J. Hussong. Raman measurements of cryogenic injection at supercritical pressure. Heat and Mass Transfer, 39, 2003.

${ }^{9}$ J.C. Oefelein and V. Yang. Modeling high-pressure mixing and combustion processes in liquid rocket engines. Journal of Propulsion and Power, 14(5):843-857, 1998.

${ }^{10}$ M. Oschwald, J.J. Smith, R. Branam, J. Hussong, A. Schik, B. Chehroudi, and D. Talley. Injection of fluids into supercritical environments. Combustion Science and Technology, 178:49-100, 2006.

${ }^{11}$ T. Schmitt, L. Selle, B. Cuenot, and T. Poinsot. Large-eddie simulation of transcritical flows. Comptes Rendus Mecanique, 337:528-538, 2009.

${ }^{12}$ R. Span, E.W. Lemmon, R.T. Jacobsen, W. Wagner, and A. Yokozeki. NIST Chemistry WebBook, NIST Standard Reference Database Number 69, chapter A Reference Equation of State for the Thermodynamic Properties of Nitrogen for Temperatures from 63.151 to $1000 \mathrm{~K}$ and Pressures to $2200 \mathrm{MPa}$. National Institute of Standards and Technology, Gaithersburg MD, 20899, http://webbook.nist.gov, (retrieved November 12, 2010)., 2010.

${ }^{13} \mathrm{~B}$. Weigand, J. Köhler, and J.v. Wolfersdorf. Thermodynamik kompakt. Springer, 2008.

${ }^{14} \mathrm{~V}$. Yang. Modeling of supercritical vaporization, mixing, and combustion processes in liquid-fueled propulsion systems. Proceedings of the Combustion Institute, 28:925-942, 2000. 